\title{
Mortality of Dandy-Walker syndrome in the United States: Analysis by race, gender, and insurance status
}

\author{
Shearwood McClelland 3rd ${ }^{1}$, Onyinyechi I. Ukwuoma ${ }^{2}$, Scott Lunos ${ }^{3}$, Kolawole S. Okuyemil ${ }^{1,4}$ \\ ${ }^{1}$ Program in Health Disparities Research, ${ }^{3}$ Biostatistics Design and Analysis Center, Clinical and Translational Science Institute, ${ }^{4}$ Department \\ of Family Medicine, University of Minnesota Medical School, Minneapolis, Minnesota, ${ }^{2}$ Department of Pediatrics, Brookdale University Hospital \\ and Medical Center, New York, USA
}

\section{ABSTRACT}

Background: Dandy-Walker syndrome (DWS) is a congenital disorder often diagnosed in early childhood. Typically manifesting with signs/symptoms of increased intracranial pressure, DWS is catastrophic unless timely neurosurgical care can be administered via cerebrospinal fluid (CSF) drainage. The rates of mortality, adverse discharge disposition (ADD), and CSF drainage in DWS may not be uniform regardless of race, gender or insurance status; such differences could reflect disparities in access to neurosurgical care. This study examines these issues on a nationwide level. Materials and Methods: The Kids' Inpatient Database spanning 1997-2003 was used for analysis. Only patients admitted for DWS (ICD-9-CM = 742.3) were included. Multivariate analysis was adjusted for several variables, including patient age, race, sex, admission type, primary payer, income, and hospital volume. Results: More than 14,0oo DWS patients were included. Increasing age predicted reduced mortality $(\mathrm{OR}=0.87 ; P<0.05), \mathrm{ADD}(\mathrm{OR}=0.96 ; P<0.05)$, and decreased likelihood of receiving CSF drainage $(\mathrm{OR}=0.86 ; P<0.0001)$. Elective admission type predicted reduced mortality $(\mathrm{OR}=0.29 ; P=0.0008)$, ADD $(\mathrm{OR}=0.68 ; P<0.05)$, and increased CSF drainage $(\mathrm{OR}=2.02 ; P<0.0001)$. African-American race $(\mathrm{OR}=1.20 ; P<0.05)$ and private insurance $(\mathrm{OR}=1.18 ; P<0.05)$ each predicted increased likelihood of receiving CSF drainage, but were not predictors of mortality or ADD. Gender, income, and hospital volume were not significant predictors of DWS outcome. Conclusion: Increasing age and elective admissions each decrease mortality and ADD associated with DWS. African-American race and private insurance status increase access to CSF drainage. These findings contradict previous literature citing African-American race as a risk factor for mortality in DWS, and emphasize the role of private insurance in obtaining access to potentially lifesaving operative care.

Key words: Congenital hydrocephalus, Dandy-Walker syndrome, healthcare disparities, mortality

\section{Introduction}

Dandy-Walker syndrome (DWS) is a congenital disorder typically manifesting clinically as hydrocephalus. The classic anatomic hallmarks of DWS are hypoplasia of the cerebellar vermis, anterior-posterior enlargement of the posterior fossa, upward displacement of the torcula and transverse sinuses, and cystic dilatation of the fourth ventricle. ${ }^{[1]}$ With an incidence of approximately 1 in 30,000,

\begin{tabular}{|l|l|}
\hline \multicolumn{2}{|c|}{ Access this article online } \\
\hline Quick Response Code: & Website: \\
\hline & www.ruralneuropractice.com \\
\cline { 2 - 2 } & \\
\hline
\end{tabular}

the clinical manifestations of hydrocephalus in DWS are diagnosed prior to the age of one in $80 \%$ of patients, and the mortality of DWS has been reported from $10 \%$ to $66 \% .^{[1-6]}$ A recent class IIB study revealed the mortality of DWS to be ten-fold higher compared with controls, and the incidence of adverse discharge disposition (ADD) to be 4.5 times higher compared with controls. ${ }^{[7]}$

The mortality of DWS is secondary to hydrocephalus, a treatable condition if the patient receives neurosurgical care via timely operative cerebrospinal fluid (CSF) drainage, which has been shown to decrease mortality in DWS by $44 \%$ and ADD by $28 \% .^{[7]}$ Consequently, examination of mortality and CSF drainage in DWS can be used as surrogate markers for access to neurosurgical care in this patient population. Two recent studies examining the New York population have indicated

\section{Address for correspondence:}

Dr. Shearwood McClelland $3^{\text {rd }}$, Program in Health Disparities Research, University of Minnesota, 717 Delaware Street SE, Suite 166, Minneapolis, Minnesota - 55414, USA. E-mail: drwood@post.harvard.edu 
significant racial disparities in postneonatal survival in DWS. ${ }^{[8,9]}$ However, the mortality of DWS on a nationwide level with a focus on potential differences related to race, gender and insurance status has yet to be examined. This study was performed to address this void.

\section{Materials and Methods}

\section{Data source}

The data source for this study was the Kids' Inpatient Database (KID) hospital discharge database (overview available at http://www.hcup-us.ahrq.gov/kidoverview. jsp) covering the years 1997 through 2003. Containing data drawn from children 20 years of age and younger, the KID was obtained from Healthcare Cost and Utilization Project, Agency for Healthcare Research and Quality (Rockville, MD). ${ }^{[10]}$ For these years, the KID contains data on $100 \%$ of discharges from a stratified random sample of nonfederal hospitals in 22 to 36 states (number of states increased year to year from 1997 to 2003), containing data on two to three million hospital discharges from a sample of 2500 to 3500 United States nonfederal hospitals.

\section{Inclusion and exclusion criteria}

The KID database was searched to identify an admission for DWS. Admissions having a patient age younger than 18 years and a diagnosis code of 742.3 (Dandy-Walker deformity or syndrome) were included.

\section{Characteristics of patients, providers, and hospitals}

In addition to race (where Caucasian was used as the reference group), patient age (for one-year increase), sex, median household income for postal (ZIP) code of residence, primary payer (Private insurance versus other), type of admission (emergency, urgent, elective, or newborn), and number of available beds (small/ medium versus large) were coded in the KID data. Furthermore, the primary procedure codes for operative CSF drainage (02.2, 02.31, 02.32, 02.33, 02.34, 02.35, 02.39, 03.71, 03.72, 03.79) were coded as well.

\section{Statistical analysis}

The characteristics of patients (inpatient stays) and hospitals were summarized by descriptive statistics. Patients admitted with DWS (ICD-9-CM = 742.3) were included for primary analysis. To examine the association between mortality/CSF drainage and the characteristics of patient age, gender, race, payer, admission type, income, and caseload of hospital in the primary analysis, we fitted multiple logistic regression models using the aforementioned characteristics as covariates. We reported odds ratios (ORs) with 95\% confidence intervals (CIs). Extrapolations to the entire U.S. population were adjusted for the NIS stratified survey method in the logistic regression models by using PROC SURVEYLOGISTIC in SAS version 9.3 (SAS Institute, Cary, NC). All $P$ values shown are two-tailed. A $P$ value less than 0.05 was deemed statistically significant. Patients were evaluated examining DWS (a) as a primary diagnosis, (b) as one of the top 3 diagnoses, and (c) as one of the top 15 diagnoses. ADD was defined as hospital discharge to any place other than home (i.e. short-term rehabilitation, long-term rehabilitation, hospice, etc.).

\section{Results}

From 1997 through 2003, the KID database contained 14,599 admissions for children with DWS.

Multivariate analysis of mortality [Table 1] revealed increasing patient age $(\mathrm{OR}=0.87 ; P=0.0024)$ and elective admission type $(\mathrm{OR}=0.29 ; P=0.0008)$ as independent predictors of reduced mortality, with newborn/other admission type $(\mathrm{OR}=3.37 ; P<0.0001)$ and "other" race $(\mathrm{OR}=1.99 ; P=0.0025)$ independently predicting increased mortality. No other variable (gender, insurance status, hospital bed size, income, African-American race, Hispanic race, Asian/Pacific Islander race, urgent admission type) proved predictive of mortality in DWS.

Multivariate analysis of ADD [Table 2] revealed increasing patient age $(\mathrm{OR}=0.96 ; P=0.0137)$ and elective admission type $(\mathrm{OR}=0.68 ; P=0.0307)$ as independent predictors of reduced ADD, with newborn/other

\section{Table 1: Multivariate analysis of mortality in DWS}

\begin{tabular}{lcc}
\hline Characteristic & OR (95\% Cl) & $P$ value \\
\hline Age (for 1 year increase) & $0.87(0.79-0.95)$ & 0.0024 \\
Female (female vs male) & $1.13(0.83-1.52)$ & 0.4388 \\
Race (White is reference group) & & \\
$\quad$ African-American & $0.89(0.61-1.29)$ & 0.5217 \\
Hispanic & $1.16(0.76-1.76)$ & 0.4996 \\
Asian or Pacific Islander & $1.11(0.50-2.45)$ & 0.7985 \\
Other & $1.99(1.27-3.10)$ & 0.0025 \\
Primary payer (private vs others) & $1.06(0.78-1.44)$ & 0.7056 \\
Admission type & & \\
(emergency is reference group) & & \\
$\quad$ Urgent & $0.85(0.49-1.46)$ & 0.5586 \\
Elective & $0.29(0.14-0.60)$ & 0.0008 \\
$\quad$ Newborn/other & $3.37(2.26-5.03)$ & $<0.0001$ \\
Large hospital bed size & $0.83(0.62-1.11)$ & 0.2105 \\
(medium/small is reference group) & & \\
Median income & & \\
(\$35,000+ is reference group) & & 0.1956 \\
1 = \$1-\$24,999 & $1.23(0.90-1.68)$ & \\
2 = \$25,000-\$34,999 & $1.24(0.80-1.91)$ & 0.3339 \\
\hline DWS: Dandy-Walker syndrome & &
\end{tabular}


admission type $(\mathrm{OR}=4.41 ; P<0.0001)$ independently predicting increased ADD. No other variable (gender, race, insurance status, urgent admission type, hospital bed size, income) proved predictive of ADD in DWS.

Multivariate analysis of CSF drainage [Table 3] revealed increasing patient age $(\mathrm{OR}=0.86 ; P<0.0001)$ as an independent predictor of reduced CSF drainage, with African-American race $(\mathrm{OR}=1.20 ; P=0.0367)$, private insurance status $(\mathrm{OR}=1.18 ; P=0.0075)$, elective admission type $(\mathrm{OR}=2.02 ; P<0.0001)$, and urgent admission

Table 2: Multivariate analysis of adverse discharge disposition in DWS

\begin{tabular}{lcc}
\hline Characteristic & OR (95\% Cl) & $P$ value \\
\hline Age (for 1 year increase) & $0.96(0.93-0.99)$ & 0.0137 \\
Female (female vs male) & $1.07(0.88-1.31)$ & 0.4758 \\
Race (White is reference group) & & \\
$\quad$ African-American & $1.15(0.92-1.45)$ & 0.2186 \\
Hispanic & $1.03(0.78-1.38)$ & 0.8198 \\
Asian or Pacific Islander & $0.86(0.41-1.78)$ & 0.6787 \\
Other & $0.96(0.67-1.38)$ & 0.8264 \\
Primary payer (private vs others) & $0.84(0.68-1.04)$ & 0.1040 \\
Admission type & & \\
(emergency is reference group) & & \\
$\quad$ Urgent & $1.10(0.86-1.42)$ & 0.4518 \\
$\quad$ Elective & $0.68(0.48-0.96)$ & 0.0307 \\
$\quad$ Newborn/other & $4.41(3.42-5.70)$ & $<0.0001$ \\
Large hospital bed size & $0.90(0.72-1.12)$ & 0.3293 \\
(medium/small is reference group) & & \\
Median income & & \\
(\$35,000+ is reference group) & & 0.1162 \\
$\quad$ 1 = \$1-\$24,999 & $1.20(0.96-1.49)$ & 0.7094 \\
\hline 2 = \$25,000-\$34,999 & $1.05(0.81-1.36)$ & \\
\hline DWS: Dandy-Walker syndrome & &
\end{tabular}

Table 3: Multivariate analysis of CSF drainage in DWS

\begin{tabular}{lcc}
\hline Characteristic & OR (95\% Cl) & $P$ value \\
\hline Age (for 1 year increase) & $0.86(0.84-0.89)$ & $<0.0001$ \\
Female (female vs male) & $1.13(0.98-1.29)$ & 0.0867 \\
Race (White is reference group) & & \\
$\quad$ African-American & $1.20(1.01-1.43)$ & 0.0367 \\
Hispanic & $1.05(0.86-1.29)$ & 0.6109 \\
Asian or Pacific Islander & $0.79(0.47-1.33)$ & 0.3799 \\
$\quad$ Other & $1.18(0.93-1.48)$ & 0.1674 \\
Primary payer (private vs others) & $1.18(1.05-1.34)$ & 0.0075 \\
Admission type & & \\
(emergency is reference group) & & \\
$\quad$ Urgent & $1.37(1.11-1.70)$ & 0.0034 \\
Elective & $2.02(1.65-2.47)$ & $<0.0001$ \\
$\quad$ Newborn/other & $0.93(0.73-1.19)$ & 0.5781 \\
Large hospital bed size & $1.06(0.89-1.26)$ & 0.5059 \\
(medium/small is reference group) & & \\
Median income & & \\
(\$35,000+is reference group) & & 0.3385 \\
$\quad$ 1 = \$1-\$24,999 & $0.93(0.80-1.08)$ & \\
2 = \$25,000-\$34,999 & $0.97(0.82-1.14)$ & 0.6833 \\
\hline CSF: Cerebrospinal fluid & &
\end{tabular}

type $(\mathrm{OR}=1.37 ; P=0.0034)$ independently predicting increased CSF drainage. No other variable (gender, Hispanic race, Asian/Pacific Islander race, other race, insurance status, newborn/other admission type, hospital bed size, income) proved predictive of CSF drainage in DWS.

\section{Discussion}

Congenital hydrocephalus is often a fatal condition unless neurosurgical intervention is expediently undertaken. Because hydrocephalus is the classic clinical manifestation of DWS, the incidence of mortality associated with DWS can serve as a surrogate marker for access to neurosurgical care in this patient population. ${ }^{[1]}$ Recent studies have indicated that racial disparities exist with regard to mortality in DWS, and that operative CSF drainage substantially reduces mortality. ${ }^{[7-9]}$ Consequently, this study was performed to evaluate the mortality of DWS on a nationwide scale and whether the incidence of mortality, ADD, or CSF drainage is impacted by race, gender or insurance status.

The findings indicate that younger age independently predicted increased mortality and ADD in DWS; the likelihood of mortality decreased exponentially per additional year of life $(P=0.0024$; odds ratio $=0.87)$. This indicates that each year of life affects mortality by $\left[\left(0.87^{\text {age }}\right.\right.$ of patient-age of comparison patient) -1$]^{*} 100$ in DWS patients. Therefore, an 8-year-old DWS patient is 13\% less likely to die than a 7-year-old patient, 50\% less likely than a 3-year-old patient, and $101 \%$ more likely than a 13 -year-old patient. Similar findings were applicable to $\operatorname{ADD}(P=0.0137$; odds ratio $=0.96$ ); that 8 -year-old DWS patient is $4 \%$ less likely than a 7-year-old patient, $18 \%$ less likely than a 3-year-old patient, and $23 \%$ more likely than a 13-year-old patient to have an ADD. These findings appear consistent with what is known about DWS and congenital hydrocephalus; most are diagnosed before the first year of life, ${ }^{[2]}$ but mortality exponentially decreases the older the child becomes. This is consistent with another finding from this study: Newborn DWS admission status independently predicted increases in both mortality and ADD. Elective admission status was also found to independently predict decreased mortality and decreased ADD in DWS, likely due to the fact that this increased the likelihood of these patients receiving early CSF drainage.

With regard to CSF drainage, while it was unsurprising to find that private insurance status independently predicted increased likelihood of receiving surgical care, it was surprising that African-American race also predicted increased likelihood of receiving neurosurgery. 
This finding, in addition to the absence of racial disparities with regard to mortality or ADD, contradicts results from previous literature citing African-American race as a risk factor for mortality in DWS. ${ }^{[8,9]}$

Despite the limitations of this study (retrospective nature, inability of ICD-9 code to distinguish hydrocephalus from etiologies other than DWS) stemming from the use of the $\mathrm{KID},{ }^{[7]}$ the results from this study indicate that with regard to DWS, access to neurosurgical care is limited by absence of private insurance status, but not by race or gender. Future studies will be needed to determine whether these findings persist over a longer period of time.

\section{References}

1. Klein O, Pierre-Kahn A, Boddaert N, Parisot D, Brunelle F. Dandy-Walker malformation: Prenatal diagnosis and prognosis. Childs Nerv Syst 2003;19:484-9.

2. Pascual-Castroviejo I, Velez A, Pascual-Pascual SI, Roche MC, Villarejo F. Dandy-Walker malformation: Analysis of 38 cases. Childs Nerv Syst 1991; 7:88-97.

3. Hirsch JF, Pierre-Kahn A, Renier D, Sainte-Rose C, Hoppe-Hirsch E. The Dandy-Walker malformation. A review of 40 cases. J Neurosurg 1984;61:515-22.
4. Long A, Moran P, Robson S. Outcome of fetal cerebral posterior fossa anomalies. Prenat Diagn 2006;26:707-710.

5. Forzano F, Mansour S, Ierullo A, Homfray T, Thilaganathan B. Posterior fossa malformation in fetuses: A report of 56 further cases and a review of the literature. Prenat Diagn 2007;27:495-501.

6. Imataka G, Yamanouchi H, Arisaka O. Dandy-Walker syndrome and chromosomal abnormalities. Congenit Anom (Kyoto) 2007;47:113-8.

7. McClelland S $3^{\text {rd }}$, Ukwuoma OI, Lunos S, Okuyemi KS. The natural history of Dandy-Walker syndrome in the United States: A population-based analysis. J Neurosci Rural Pract 2015;6:23-6.

8. Salihu HM, Kornosky JL, Druschel CM. Dandy-Walker syndrome, associated anomalies and survival through infancy: A population-based study. Fetal Diagn Ther 2008;24:155-60.

9. Salihu HM, Kornosky JL, Alio AP, Druschel CM. Racial disparities in mortality among infants with Dandy-Walker syndrome. J Natl Med Assoc 2009;101:456-61.

10. Steiner C, Elixhauser A, Schnaier J. The healthcare cost and utilization project: An overview. Eff Clin Pract 2002;5:143-51.

How to cite this article: McClelland S, Ukwuoma OI, Lunos S, Okuyemi KS. Mortality of Dandy-Walker syndrome in the United States: Analysis by race, gender, and insurance status. J Neurosci Rural Pract 2015;6:182-5.

Source of Support: Funding for statistical analysis was provided by the Department of Family Medicine and Community Health, University of Minnesota Medical School (S.L.). No other author received financial support in conjunction with the generation of this manuscript. Dr. McClelland had full access to all of the data in the study and takes responsibility for the integrity of the data and the accuracy of the data analysis. Conflict of Interest: None declared.

\section{New features on the journal's website}

\section{Optimized content for mobile and hand-held devices}

HTML pages have been optimized of mobile and other hand-held devices (such as iPad, Kindle, iPod) for faster browsing speed.

Click on [Mobile Full text] from Table of Contents page.

This is simple HTML version for faster download on mobiles (if viewed on desktop, it will be automatically redirected to full HTML version)

\section{E-Pub for hand-held devices}

EPUB is an open e-book standard recommended by The International Digital Publishing Forum which is designed for reflowable content i.e. the text display can be optimized for a particular display device.

Click on [EPub] from Table of Contents page.

There are various e-Pub readers such as for Windows: Digital Editions, OS X: Calibre/Bookworm, iPhone/iPod Touch/iPad: Stanza, and Linux: Calibre/Bookworm.

\section{E-Book for desktop}

One can also see the entire issue as printed here in a 'flip book' version on desktops. Links are available from Current Issue as well as Archives pages.

Click on View as eBook 